\title{
The radiologist's role in pathologic diagnosis of small lung nodules: radiographic methods of tissue acquisition
}

\author{
Matthew D. Cham ${ }^{1}$, Claudia I. Henschke ${ }^{2,3}$, David F. Yankelevitz ${ }^{2}$ \\ ${ }^{1}$ Department of Radiology, University of Washington Medical Center, Seattle, WA, USA; ${ }^{2}$ Department of Radiology, Icahn School of Medicine, \\ Mount Sinai Medical Center, New York, NY, USA; ${ }^{3}$ Department of Radiology, Phoenix VA Health Care System, Phoenix, AZ, USA \\ Contributions: (I) Conception and design: All authors; (II) Administrative support: All authors; (III) Provision of study materials or patients: None; \\ (IV) Collection and assembly of data: None; (V) Data analysis and interpretation: None; (VI) Manuscript writing: All authors; (VII) Final approval of \\ manuscript: All authors. \\ Correspondence to: Matthew D. Cham, MD. Professor of Radiology, $700234^{\text {th }}$ Ave NE, Seattle, WA 98115, USA. Email: mdcham@uw.edu.
}

\begin{abstract}
Over the past decade, chest computed tomography (CT) utilization has steadily increased in conjunction with lung cancer CT screening. As a result, small lung nodules have become an increasingly common finding. CT-guided lung biopsy plays a pivotal role in the sampling of these small lung nodules and the subsequent diagnosis of lung cancer. The success of lung biopsies has a substantial downstream impact on diagnosis and treatment planning. It is well-recognized that the efficacy of lung biopsy is both operator-dependent and patient-dependent. Several considerations are necessary for the safe and successful performance of CT-guided lung biopsy. A familiarity with biopsy contraindications and their management can drastically reduce serious complications, such as intractable hemorrhage. Conscientious patient preparation and empathic communication are essential for reducing patient anxiety while increasing patient tolerance for the procedure and its common complications. Strategic and precise nodule-targeting techniques are crucial for obtaining adequate tissue and for improving diagnostic accuracy. On-site cytologic assessment is a powerful tool that can further boost diagnostic yield while also reducing complication rates by eliminating the need for additional biopsy attempts. Vigilant observation and careful assessment throughout the procedure are key factors in the effective mitigation of the most frequently encountered complications. As personalized medicine plays an increasingly integral part in the diagnosis and treatment of early-stage lung cancers, coordination between radiologists, clinicians, pathologists, and the patient becomes especially important.
\end{abstract}

Keywords: Lung biopsy; lung nodule biopsy; CT-guided lung biopsy; lung biopsy techniques; lung biopsy complications

Received: 05 September 2020; Accepted: 26 July 2021; Published: 25 November 2022.

doi: $10.21037 /$ ccts-21-36

View this article at: https://dx.doi.org/10.21037/ccts-21-36

\section{Introduction}

The widespread use of computed tomography (CT) has increased the frequency of detecting small indeterminate pulmonary nodules. Traditional symptom-detected lung cancers are frequently greater than $2 \mathrm{~cm}$ in size and are easily visible on chest radiographs. On the other hand, today's CT-detected lung cancers are often smaller than
$2 \mathrm{~cm}$ and, in some cases, may even be challenging to detect on chest CT. In the healthy asymptomatic lung cancer screening CT population, the judicious use of minimally invasive diagnostic techniques is particularly relevant in order to minimize harm. Many strategies have been developed to improve the technical success rate, safety, and diagnostic accuracy of CT-guided lung biopsy in the workup of small indeterminate nodules. 


\section{Indications for lung biopsy}

The indications for lung biopsy in the setting of CT screening include lung nodules greater than or equal to 15 $\mathrm{mm}$ that do not resolve with antibiotics or lung nodules less than $15 \mathrm{~mm}$ that have a documented growth or are FDG-avid during PET-CT $(1,2)$. For lung nodules that are incidentally detected during CT scans, Fleischner Society guidelines advocate the consideration of lung biopsy for solid lung nodules that are greater than $8 \mathrm{~mm}$ and have a documented growth (3). This guideline does not apply to patients with known cancer who may require a more aggressive workup or staging exam. It also does not apply to incidental nodules in patients younger than 35 years of age who are much less likely to have a primary lung malignancy.

\section{Lung biopsy in CT screening}

In the context of lung cancer CT screening, the frequency of biopsies is around 2\% during the baseline round and around $0.5 \%$ during subsequent rounds. This means that approximately $2 \%$ of subjects will require biopsy of a suspicious nodule on the first CT scan, while $0.5 \%$ of subjects will require biopsy of a nodule based on each subsequent round of screening. There is a substantial amount of data derived from the first 10 years of CT screening. In a large cohort study, among 31,567 asymptomatic participants undergoing baseline screening, 405 were found to have lung cancer on their baseline CT. Among 27,456 annual screenings, 74 were found to have lung cancer during their annual screening CT. In total, 484 received a diagnosis of lung cancer and 412 had a clinical stage I lung cancer (4).

It is widely recognized that CT screening detects much smaller lung nodules compared to symptom-prompted imaging. The screening time point affects the likelihood of needing a lung biopsy as well as the nodule size distribution. Among 4,782 subjects screened during a 6-year period in the Princess Margaret Hospital (Toronto, Canada) lung cancer CT screening program, 128 lung nodules were biopsied (5). Seventy-nine of 128 (62\%) biopsies occurred within the first year of CT screening, 22 of 128 (17\%) biopsies occurred between years 1 and 2, and 27 of 128 (21\%) biopsies occurred between years 2 to 6 . Furthermore, the nodule size was considerably larger for years 0 to 1 compared to those from years 1 to 6 . The vast majority (81\%) of lung nodules biopsied during years 0 to 1 had no growth information and had a larger mean diameter, greater than $15 \mathrm{~mm}$, at the time of presentation. On the other hand, lung nodules biopsied during years 1 to 6 had a smaller mean diameter and were either new $(31 \%)$ or had a documented growth (69\%).

\section{Contraindications}

There are only a few contraindications for CT-guided lung biopsy. The most relevant absolute contraindication is an uncooperative patient. Patients who are uncooperative or combative will likely decrease the technical success rate of the procedure and the level of patient safety. Relative contraindications include a platelet count of less than 50,000 , an international normalized ratio (INR) greater than 1.5 , severe bullous emphysema, or contralateral pneumonectomy. If platelet count and INR cannot be corrected due to underlying comorbidities, lung biopsy should be avoided due to the risk of intractable pulmonary hemorrhage and death. In patients with severely reduced pulmonary reserve, such as those with severe bullous emphysema or contralateral pneumonectomy, a thoracic surgeon should be notified prior to the procedure and be immediately available in the event that assistance may be needed for management and possible emergent chest tube placement.

\section{Pre-biopsy communication}

Proper patient communication is essential prior to the biopsy procedure. One week before the procedure, it is important to discuss the risks, benefits, and alternatives with the patient. Five days prior to the procedure, anticoagulants and antiplatelet medications should be discontinued. There are a few rare exceptions, such as patients requiring continued anticoagulation for specific implanted devices. These cases would necessitate discussion with the primary care team. One day prior to the procedure, the platelet count and INR must be re-checked to ensure patient safety. Guidance regarding specific medications is available from several societies $(6,7)$.

Sedation is generally not recommended during lung biopsy as it can reduce the level of patient cooperation (7). Patients who are somnolent during the procedure are more likely to engage in sudden involuntary movements that increase the risk of injury during the procedure. In rare cases when patients have CT scanner claustrophobia, alprazolam $0.25 \mathrm{mg}$ by mouth or midazolam $1 \mathrm{mg}$ by intravenous injection may be considered. 

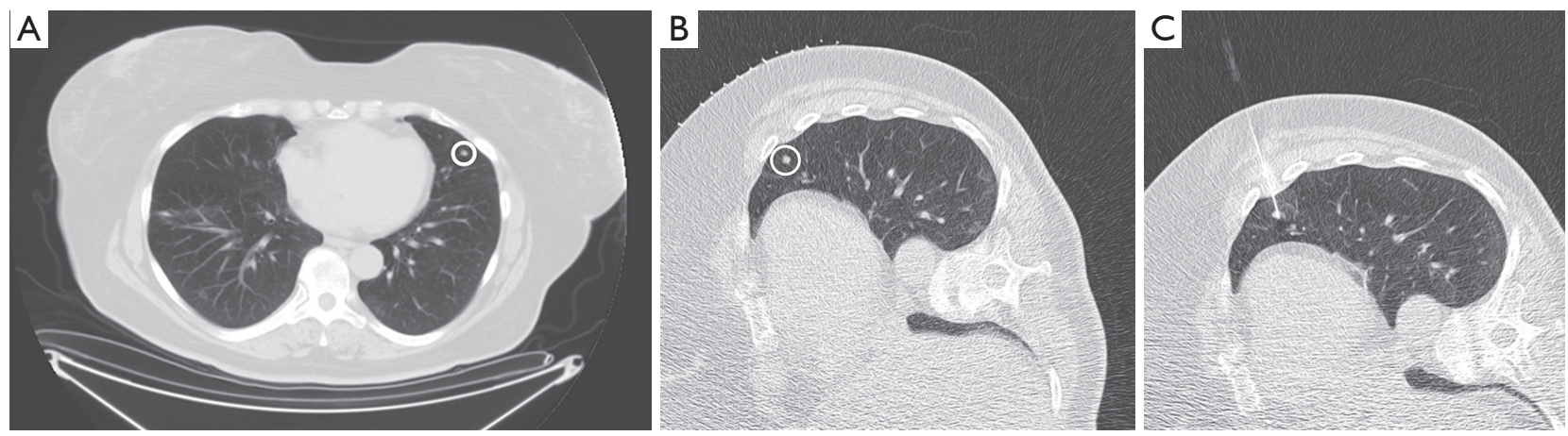

Figure 1 Repositioning. (A) Supine position with small left upper lobe nodule located under several centimeters of breast tissue; (B) decubitus view demonstrates shift of soft tissue with easier access; $(\mathrm{C})$ needle tip within the nodule. The white circles indicate the lung nodule.

It is important for patients to take gentle breaths throughout the procedure. For lung nodules that are near the diaphragm, respiratory suspension during image acquisition and needle insertion may allow for greater success in lung nodule targeting. In these cases, preprocedural breathing instructions and coaching of the patient is necessary to ensure proper patient cooperation.

On the day of the lung biopsy, it is important to notify patients regarding details of the procedure. Patients must be reminded to lie very still on the CT scanner throughout the procedure even though the CT table can be quite firm and uncomfortable. Patients who are surprised by sensations arising from needle manipulation or are unprepared for the duration of the procedure are more likely to stop cooperating and to move during the procedure, which may decrease technical success, diagnostic accuracy, and patient safety.

\section{Biopsy planning}

When planning the biopsy, the ideal needle path is determined by anatomic obstacles, fissures along the path, and the depth of the lung nodule. A supine, prone, or decubitus position may be necessary to avoid obstacles like lung fissures which would increase complication rates when traversed (Figure 1). After the appropriate needle path is selected, $1 \%$ lidocaine is administered subcutaneously and at the pleura. Special care must be taken to avoid inadvertently puncturing the pleura and causing a pneumothorax during lidocaine administration. It is often possible to feel when the needle tip reaches the pleura and sufficient lidocaine can be administered at this point since the parietal pleura contains pain fibers.
There are 2 major types of lung biopsy needles: a fine needle aspiration (FNA) needle and a core biopsy needle. A FNA needle is typically a single shaft needle measuring 22-23 gauge, although they can be coaxial (needle-withina-needle). A core biopsy needle is generally larger in caliber, measuring 18-21 gauge, and involves the use of an introducer needle as part of a coaxial system. The main advantage of a core biopsy needle is its ability to obtain multiple samples and larger histologic samples via a single pleural puncture, at the risk of an increased complication rate resulting from its larger needle gauge. On the other hand, the single shaft FNA needle is associated with up to $70 \%$ lower complication rates owing to its smaller needle gauge, despite requiring potentially multiple pleural punctures given its lack of an introducer needle (8).

Multiple pleural punctures can theoretically increase the rate of complications such as a pneumothorax. However, numerous studies have not found a significant correlation between the number of pleural punctures and the pneumothorax rate (9-19). On the other hand, the larger gauge of core biopsy needles has been associated with a significantly higher rate of complications including pneumothorax, pulmonary hemorrhage, and hemoptysis $(20,21)$. One large retrospective study found a statistically similar but clinically lower incidence of pneumothorax when using non-coaxial needles $(77 / 332$ or $23 \%)$ compared to coaxial needles $(86 / 318$ or $27 \%)(\mathrm{P}=0.14)(22)$. The same investigators found a similar incidence of pulmonary hemorrhage when using non-coaxial needles $(65 / 332$ or $20 \%$ ) compared to coaxial needles ( $71 / 318$ or $22 \%)$, which was not a statistically significant difference $(\mathrm{P}=0.27)(23)$.

In a 2004 survey of practice patterns involving Society of Thoracic Radiology members, about half of surveyed 
radiologists preferred single shaft FNA needles (24). With the advent of molecular analysis and personalized medicine, there has been a gradual shift towards using both coaxial and FNA techniques during each biopsy procedure, with some studies demonstrating that combined FNA/core biopsy increases diagnostic accuracy over either FNA or core biopsy alone (25). In a 2017 survey of practice patterns again involving Society of Thoracic Radiology members, about $43 \%$ of thoracic radiologists preferred combined FNA/core, $42 \%$ preferred core biopsy alone, and $15 \%$ preferred FNA alone (26).

\section{Procedural technique}

During the lung biopsy, a low-dose CT technique is typically used. This includes a tube voltage of $120 \mathrm{kVp}$ or less and tube current ranging from 10-40 mAs depending on body habitus. This results in an estimated radiation dose of less than $1 \mathrm{mSv}$. In general, a noisy image does not impact the ability to perform the procedure. It is very important to document and confirm that the needle tip is within the nodule during every biopsy. This should be performed using a thin 1-2 $\mathrm{mm}$ slice thickness to prevent falsenegative results arising from partial volume averaging (27). In general, it is useful to document that images above and below the needle tip still contain the nodule.

Nodule targeting is a critical determinant of technical success during lung biopsy. A seemingly trivial 3-degree deviation can cause the needle to miss a $1 \mathrm{~cm}$ nodule that is $10 \mathrm{~cm}$ deep. A well-established nodule targeting method used in CT-guided FNA is knowns as the "bevel steering" technique (28). During lung biopsy, it is important to be aware of the direction that the needle bevel is facing, which is indicated on the needle hub by a visible marker. Bevel steering was specifically developed to successfully direct the needle tip towards small targets. In bevel steering, the proceduralist rotates the needle such that the beveled edge faces away from the intended trajectory. For example, if the needle must be directed medially to reach a nodule, then the bevel must be rotated until it faces laterally. This needle rotation is performed prior to advancing a needle that is already within the chest wall or lung parenchyma (Figure 2). This bevel positioning has the effect of incrementally redirecting the needle as it is being advanced deeper into lung parenchyma. The bevel face can be rotated multiple times within a single needle pass, to redirect the needle towards a small or moving nodule. The bevel steering technique allows the proceduralist to maneuver the needle tip in any direction, including medial, lateral, cranial, or caudal directions and anywhere in between (Figure 3). An additional way this technique can be used has been termed "vessel steering". In this situation the same approach to guide the needle is used to avoid blood vessels. This allows for lesions to be safely biopsied and avoid hemorrhage (Figure 4). The ability to accurately steer the needle during the course of the procedure allows for a safer procedure and also allows for access to virtually any lesion within the chest, including small cystic or cavitary lesions where the needle must be placed within the wall (Figure 5).

\section{Diagnostic accuracy}

The diagnostic accuracy of CT-guided biopsy is highly dependent on nodule size and location, operator expertise, and cytologic expertise. In general, the accuracy for malignant nodules is approximately $95 \%$, the accuracy for benign nodules is around $80 \%$, and the accuracy for lung nodules less than $1.5 \mathrm{~cm}$ is between $74-95 \%$. The overall accuracy reported by meta-analyses is in the order of 89 97\% (29-32).

For small lung nodules, the diagnostic accuracy increases with increasing nodule size. In a small retrospective study of 43 FNA biopsies of sub- $8 \mathrm{~mm}$ nodules, Suresh et al. found a diagnostic accuracy of $81.4 \%$ and sensitivity of $88.9 \%$, significantly higher than those of PET-CT alone which had an accuracy of $46.5 \%$ and sensitivity of $38.9 \%$, $\mathrm{P}<0.001$ (33). In another retrospective review of 117 core biopsies of nodules measuring $8 \mathrm{~mm}$ or less, Zhao et al. reported a higher diagnostic accuracy, sensitivity, and specificity of $95.7 \%, 95.8 \%$, and $95.5 \%$ respectively (34). In a retrospective study involving 94 core biopsies of lung nodules measuring $8 \mathrm{~mm}$ or less, Chang et al. reported a diagnostic accuracy, sensitivity, and specificity of $90.4 \%$, $87.1 \%$, and $100 \%$ respectively; sub- $6 \mathrm{~mm}$ nodules had a significantly higher nondiagnostic rate of $15 \%$ compared to $4 \%$ for nodules between 8 to $10 \mathrm{~mm}$ in size $(\mathrm{P}=0.017)(35)$. In another retrospective study of 101 core biopsies of slightly larger lung nodules measuring $10 \mathrm{~mm}$ or less, Li et al. found a diagnostic accuracy, sensitivity, and specificity of $90.0 \%, 80 \%$, and $100 \%$ respectively, with hemoptysis and small sample count independently predictive of diagnostic failure in multivariate analysis (36). In a retrospective study of 198 core biopsies, Huang et al. reported a reduced diagnostic accuracy of $83.7 \%$ for nodules $<15 \mathrm{~mm}$ compared to $96.8 \%$ for nodules $\geq 15 \mathrm{~mm}$ (37). Chen et al. performed a retrospective review of 168 core biopsies of larger nodules 

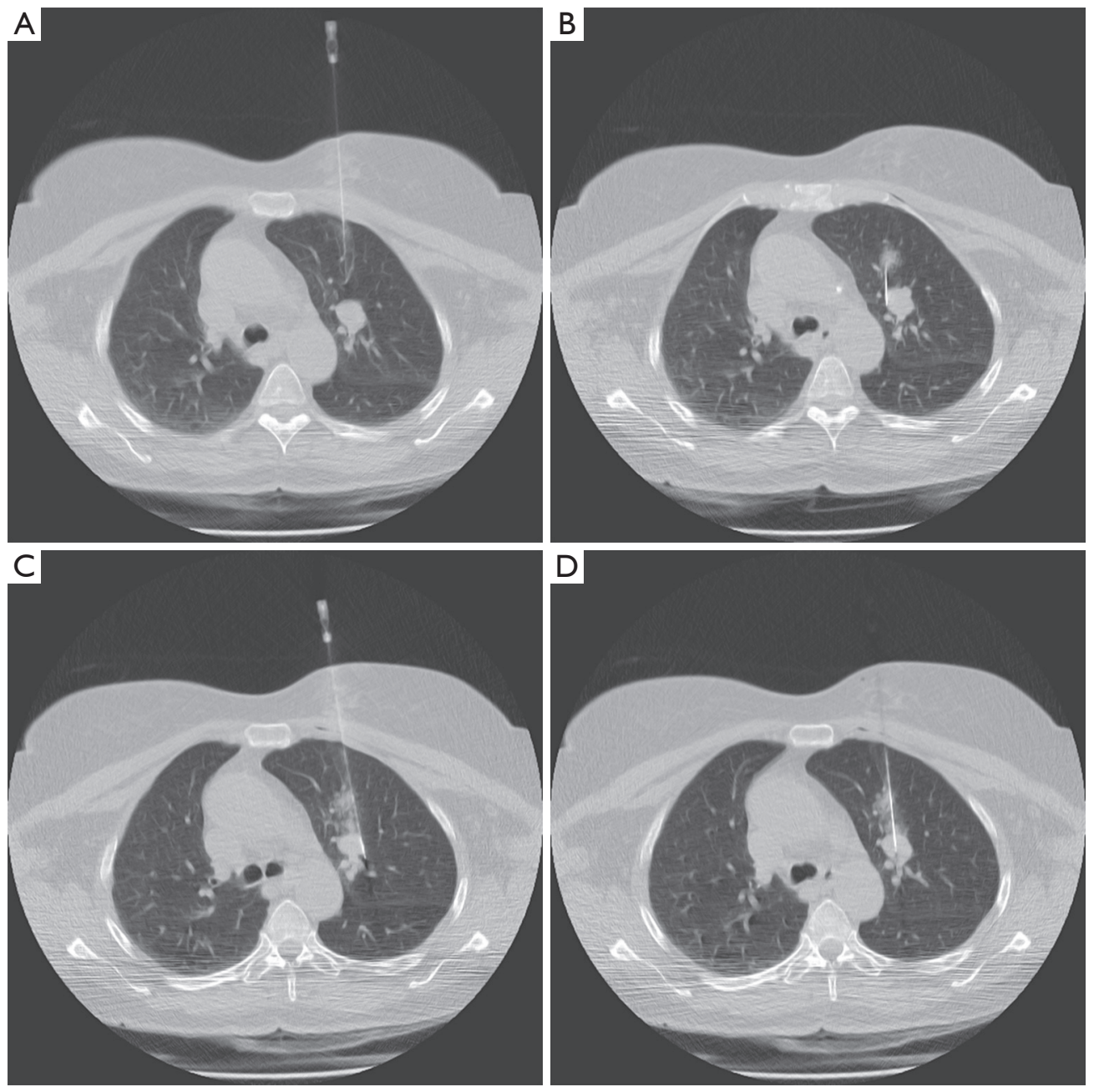

Figure 2 Bevel steering. (A) Needle appears to be on target towards center of left upper lobe nodule; (B) upon advancing the needle, the tip is now on the medial edge of the nodule. Patient may have moved slightly and caused the change in trajectory; (C) needle is partially withdrawn bevel turned in opposite direction from the nodule, torque applied to steer laterally, and re-advanced. However, its location was changed too much and it is now on the lateral edge of the nodule and again not in optimal location to obtain a sample. Note that the needle was moved the entire width of the nodule; (D) second repositioning now shows needle tip within the center of the nodule. In this instance, the needle was again partially withdrawn, the bevel turned away from the nodule and torque was applied to the shaft to steer it medially. The amount of torque was less than the first repositioning so that the amount of movement was less.

measuring $20 \mathrm{~mm}$ or less, and found a similar diagnostic accuracy, sensitivity, and specificity of $92.2 \%, 91.0 \%$, and $100 \%$ (38). Diagnostic failure and false negative rates also increase with large nodules that measure more than $27 \mathrm{~mm}$ in diameter, likely due to sampling error from adjacent atelectasis or central necrosis $(39,40)$. These large nodules and lung masses are beyond the scope of this chapter.

Nodule consistency also affects diagnostic accuracy. Nonsolid nodules may be more difficult to biopsy because they are easily obscured by mild parenchymal hemorrhage during the procedure. In a small study evaluating FNA of 32 subsolid (part-solid and nonsolid) nodules, sensitivity, specificity, and positive predictive value of $89.2 \%, 100 \%$, and $100 \%$ were reported (41). In a retrospective study of 198 FNA's, De Filippo reported a diagnostic accuracy of $95.1 \%$ for solid nodules, $84.6 \%$ for part-solid nodules, and only $66 \%$ for nonsolid nodules, $\mathrm{P}<0.05$ (42). In a large retrospective study of 354 consecutive core biopsies, Yun et al. found no significant difference in diagnostic accuracy, sensitivity, specificity, nondiagnostic rate, and 

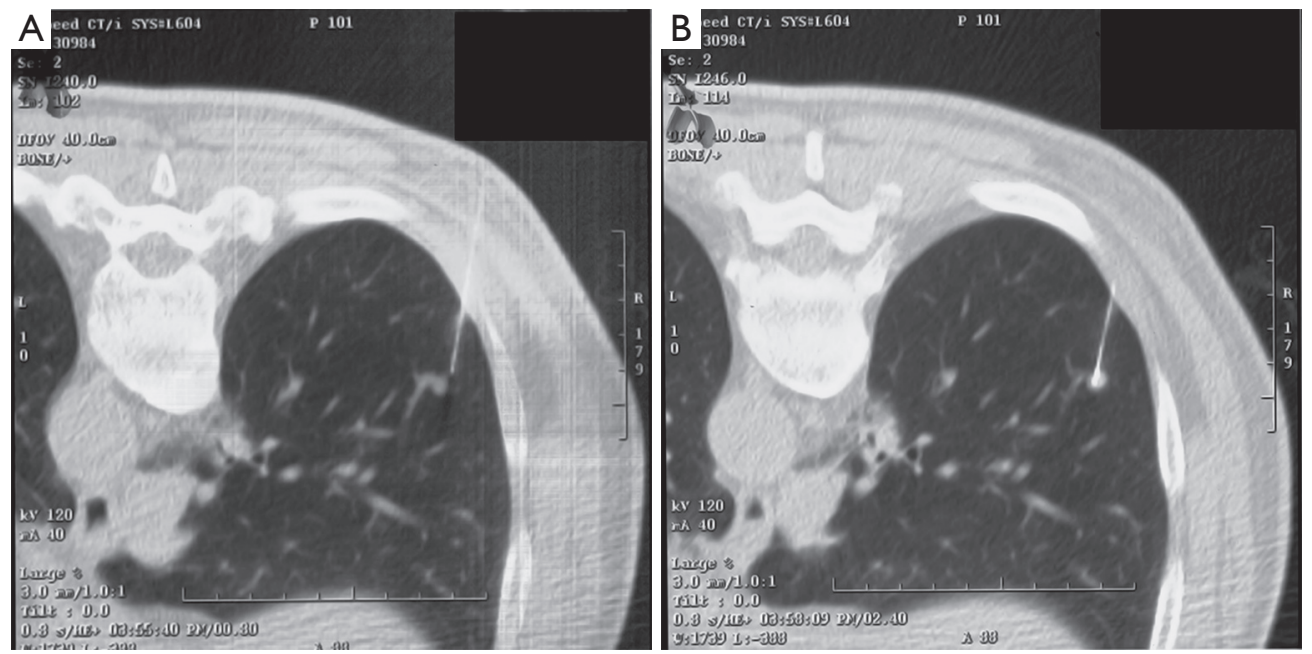

Figure 3 Bevel steering. (A) Needle tip is on course to miss the nodule being laterally displaced; (B) bevel turned away from the nodule, needle pulled back partially and re-advanced with gentle torque applied to the needle. It now enters the center of the nodule.
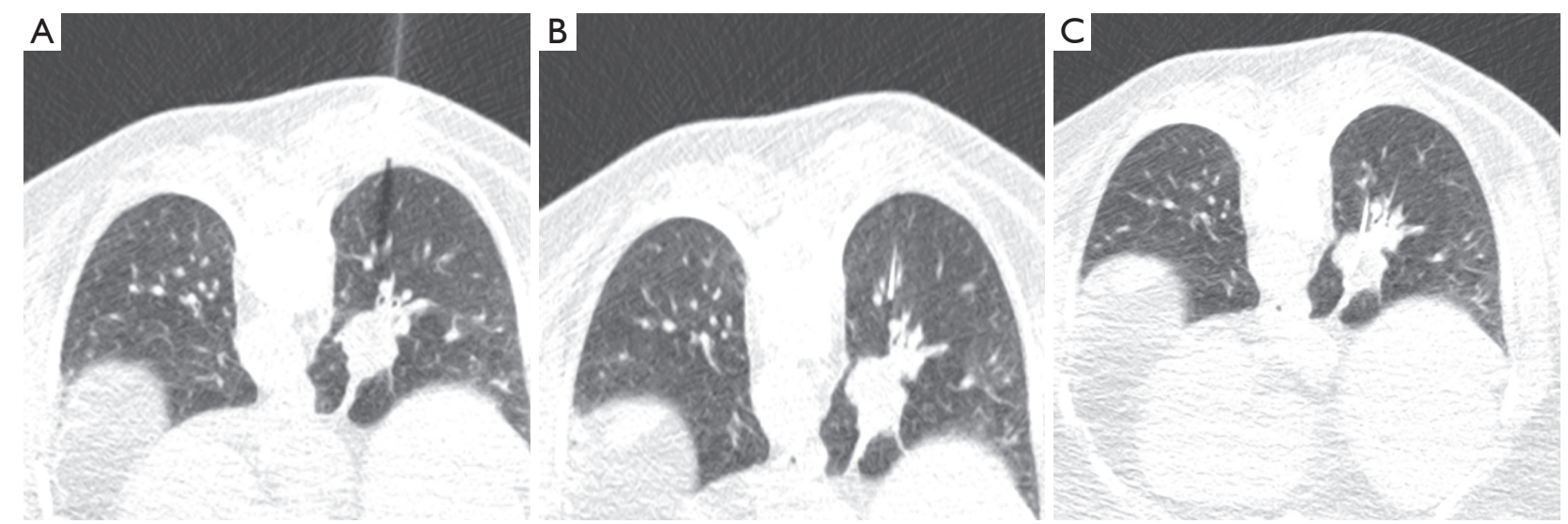

Figure 4 Vessel steering. (A) Nodule located deep in the right lower lobe with multiple small vessels located in front of it in the potential needle path; (B) needle guided between the first two vessels. However, it is on course to hit a vessel located deeper; (C) using the technique of bevel steering, the needle can be maneuvered medially to miss the vessel and enter the nodule. In this manner we minimize the chances of bleeding.

pneumothorax rate between solid versus part-solid nodule biopsies $(\mathrm{P}>0.8)$ (43). These contradictory results are likely due to confounding variables including needle type. In a retrospective study comparing the diagnostic accuracy of FNA versus core biopsy in consecutive subsolid nodules measuring $25 \pm 14 \mathrm{~mm}$, core biopsy was found to alter the FNA diagnosis in $4.8 \%$ of cases (44).

Several other factors may indirectly affect diagnostic accuracy because of their effect on pneumothorax rates which can prematurely terminate the procedure. Ohno et al. reported that a needle path length of $<40 \mathrm{~mm}$ had a diagnostic accuracy of $>88 \%$ whereas a needle path length $>40 \mathrm{~mm}$ had a reduced accuracy of $<62 \%(45)$. Ko et al. reported that a needle-pleural angle $<80$-degree, decreased forced expiratory volume in 1 second to vital capacity ratio of $<50 \%$, lateral pleural punctures, and perifissural nodule location significantly increase pneumothorax rates $(\mathrm{P}<0.05)(12)$. Nakamura et al. reported that a prone position significantly reduces the pneumothorax rate $(\mathrm{P}=0.026)(46)$. Emphysema severity is another well-established risk factor for pneumothorax development during lung biopsy (47).

In the setting of a nondiagnostic biopsy result, the next 

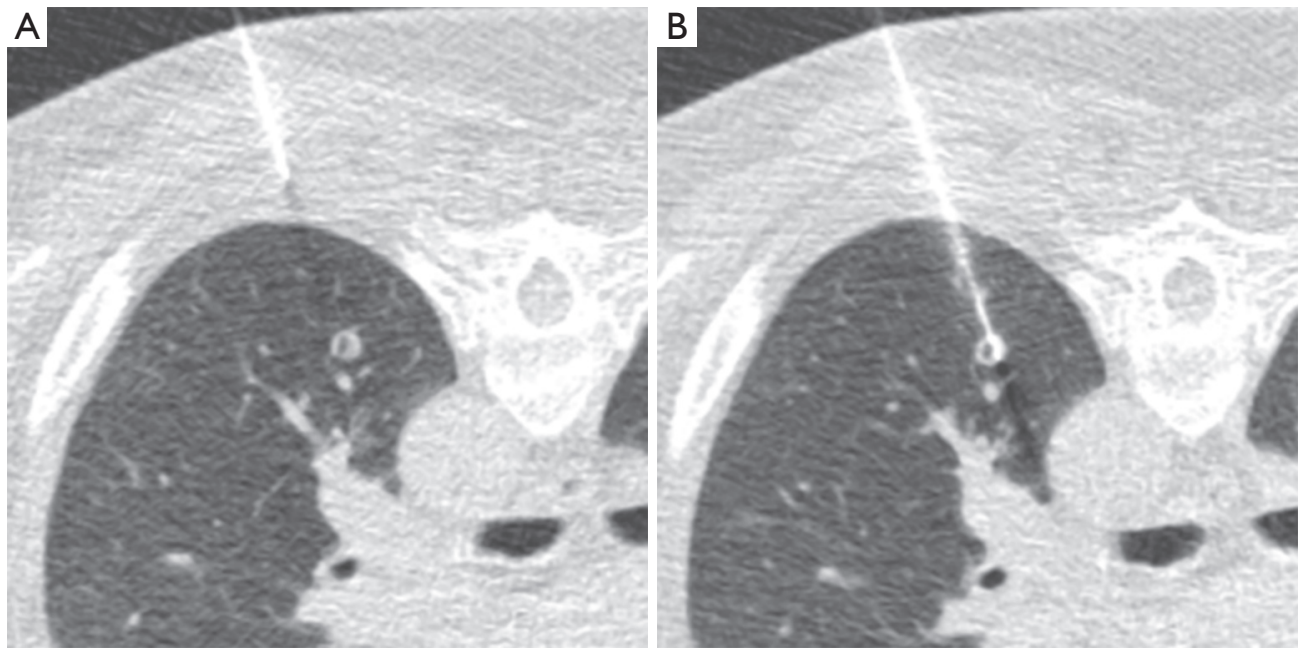

Figure 5 Cystic lesion. (A) Small cystic lesion located in left lower lobe; (B) using bevel steering the needle is directed towards the wall of the cystic lesion where viable tissue remains.

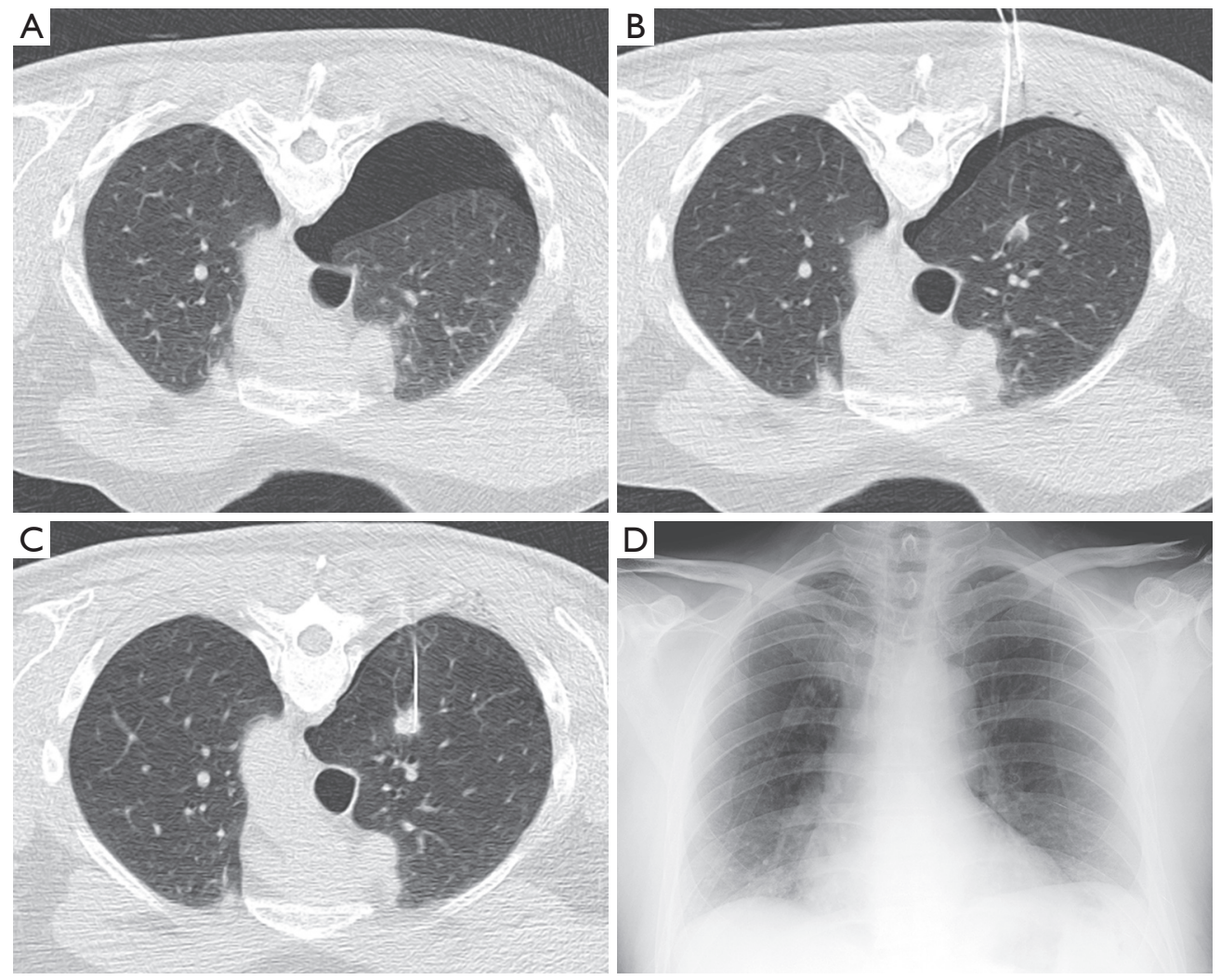

Figure 6 Pneumothorax aspiration. (A) A moderate pneumothorax developed after local anesthesia was delivered prior to insertion of the biopsy needle; (B) a catheter was placed, connected to a three-way stop cock and the pneumothorax was drained. The biopsy needle (lateral) was placed into the soft tissues; (C) the pneumothorax needed to be drained multiple times during the performance of the procedure. The biopsy needle was advanced into the lesion between pneumothorax aspirations; (D) chest radiograph one hour post procedure showed no evidence for residual pneumothorax. 
steps in management include repeat imaging or repeat tissue sampling. Repeat imaging may be acceptable for lesions that have a nonspecific benign result on pathology. On the other hand, atypical cells, nodule growth, or suspicious imaging features may warrant repeat tissue sampling due to their increased likelihood of malignancy. In one of the largest retrospective studies to date, Lee et al. found that $53.7 \%$ of patients who underwent repeat CT-guided biopsy after an initial nondiagnostic result obtained a definitive diagnosis from the second biopsy (48). They also found that $100 \%$ (130/130) of atypical lesions suggestive of malignancy were ultimately found to be malignant.

\section{Complications and mitigation strategies}

The most common complication during lung biopsy is a pneumothorax. The pneumothorax rate is approximately $25 \%$, and is affected by chronic obstructive pulmonary disease (COPD) severity, depth of lung nodule, size of nodule, needle trajectory, and needle size. The vast majority of these pneumothoraces are trace pneumothoraces that are too small to warrant chest tube placement. A chest tube is required in approximately $2-5 \%$ of FNA biopsies and is typically placed when the pneumothorax is either symptomatic or moderate in size (greater than $30 \%$ in volume). A thoracic vent is a special chest tube that can be placed by the radiologist at the bedside. The thoracic vent contains a one-way valve that allows the pneumothorax to be expelled as the patient breathes, thus relieving the pneumothorax.

The rate of chest tube placement can also be reduced using several techniques such as manual aspiration of pneumothorax (49). During manual aspiration, an 18-gauge Angiocath, or the equivalent such as a Cook catheter with multiple side holes, can be advanced into the pleural space. Using an attached IV tubing, three-way stopcock, and $60 \mathrm{~mL}$ syringe, the pneumothorax can be completely and permanently aspirated in some cases (Figure 6). This manual aspiration technique may not work for pneumothoraces that are greater than $670 \mathrm{~mL}$ (50). Manual aspiration has been shown to reduce the chest tube placement rate by half. In patients who are asymptomatic despite having a moderatesized stable pneumothorax, another option is to admit the patient to the hospital for overnight monitoring, thereby reducing the need for chest tube placement.

Other techniques for reducing the rate of chest tube placement include the blood patch technique and the injection of chemical sealants into the needle tract. These techniques require the use of a coaxial needle system that incorporates an introducer needle. In the blood patch technique, $10 \mathrm{~mL}$ of autologous blood is drawn from a peripheral vein prior to the procedure. After the biopsy is completed, the collected autologous blood is injected into the introducer needle as the introducer needle is withdrawn, thereby filling and sealing the needle tract with coagulated blood. The blood patch technique has been found to significantly reduce the rate of pneumothorax by 30-70\% (51-53). The usefulness of this technique is reduced in patients with bullous emphysema where there may be no discrete needle tract for the coagulated blood to occupy. Some authors have also reported success using 1 to $3 \mathrm{~mL}$ of normal saline in lieu of autologous blood as a sealant (54).

Similar to the blood patch technique, specially developed chemical sealants can be injected into the introducer needle as the needle is withdrawn, thereby sealing the needle tract and preventing a pneumothorax $(55,56)$. Some of the reported complications from commercial sealants include pain at the sealant site and chronic FDG-uptake related to pleural inflammation which could be mistaken for malignancy or disease recurrence during PET-CT. In one prospective randomized controlled trial, the rate of postbiopsy pneumothorax was clinically lower but statistically similar when using an autologous blood patch (42/199 or $21 \%)$ compared to a hydrogel plug $(60 / 208$ or $29 \%)$ $(\mathrm{P}=0.07)(57)$.

In a 2017 survey of practice patterns involving Society of Thoracic Radiology members, 32\% of thoracic radiologists utilized intraprocedural preventive measures to minimize the risk of pneumothorax (26).

\section{Other complications}

Another frequent complication during lung biopsy is hemoptysis which occurs in approximately $5 \%$ of patients. In patients with normal coagulation parameters, hemoptysis is typically self-limited. Care must be taken to avoid traversing vascular structures during lung biopsy, including vessels along the chest wall such as the internal mammary arteries. Less frequent complications include hemothorax, air embolism, seeding of the biopsy tract, hemopericardium, and lung torsion. Most of these rare complications are much less likely to occur when using a single shaft fine needle aspiration technique, as opposed to a coaxial core biopsy technique. Systemic air embolism, although rare, can be a fatal complication if not recognized or treated promptly. For a venous air embolism, the typical management includes 
Trendelenberg with left lateral decubitus positioning (to confine air emboli within the left atrium), supplemental oxygen administration, intravenous fluid administration, and blood pressure support (58).

The complication rate for lung biopsies is also highly operator dependent. In the literature, the pneumothorax rate is about $20-25 \%$ while the chest tube rate is about 4-6\% (8). At our institution, a review of one radiologist's complication rate during 211 consecutive CT-guided lung biopsies revealed a pneumothorax rate of only $5.2 \%$ and a chest tube rate of only $0.47 \%$, underscoring the substantial operator dependence of this procedure including its complication rates.

\section{Discharge planning}

A follow-up expiratory chest radiograph is routinely performed 1 to 2 hours after lung biopsy to evaluate for a pneumothorax. If there is a known pneumothorax based on the CT images acquired during biopsy, then the chest radiograph can be performed 1 hour after the biopsy. In the absence of a pneumothorax on chest radiography, outpatients may be discharged home with instructions (see next paragraph) (59). If a small pneumothorax is present on the chest radiograph, then another chest radiograph is obtained 2 to 3 hours after the biopsy to evaluate for pneumothorax stability. If a small and stable pneumothorax is present in the absence of symptoms, then outpatients may be safely discharged home with instructions. If the pneumothorax is moderate in size or small but worsening, then patients may be admitted to the hospital for overnight observation. Frail or elderly patients who live alone or have no at-home caregiver may also be admitted for overnight observation.

Patients are provided with several discharge instructions, including holding anticoagulants and NSAIDs for 5 days, avoiding air travel for 2 weeks, and avoiding strenuous activities for 3 days. Patient are also notified about the possibility of a delayed pneumothorax which may develop 24 to 48 hours after discharge. The probability of a delayed pneumothorax developing more than 24 hours after lung biopsy is about 1 to $3 \%$ (60-63). All patients are instructed to proceed to the nearest emergency room if they experience increasing shortness of breath or increasing chest pain.

\section{On-site cytology}

Cytology is a critical component of lung biopsy. It is exceedingly helpful to have a cytologist or cytotechnologist present during the biopsy and to review the specimen for adequacy immediately after tissue acquisition. This workflow has been referred to as rapid on-site evaluation (ROSE) or immediate cytologic evaluation (ICE). In this workflow, the specimens are quick stained, air-dried, and evaluated under a microscope to determine whether additional samples are necessary. Immediate feedback given to the radiologist will help direct radiologist. For example, if the sample is deemed adequate after the first pass, then additional pleural punctures or passes will not be necessary, thus reducing potential complications. If on the other hand, the sample contains predominantly necrotic material, then the radiologist can direct the needle towards the periphery of the nodule, thereby avoiding areas of central necrosis. If the sample contains predominantly inflammatory cells, then the radiologist can redirect the needle towards the center of the nodule, thereby avoiding the reactive cells that may encase a malignant tumor. Several prospective studies have found that rapid on-site evaluation significantly increases the diagnostic accuracy of CT-guided lung biopsy (9,64-66). In a 2017 survey of practice patterns involving Society of Thoracic Radiology members, $71 \%$ of thoracic radiologists reported having rapid on-site cytology at their institution (26).

\section{Biomarkers and personalized medicine}

Molecular markers have become increasingly important in the era of personalized medicine (67). Lung cancers with certain biomarkers have been found to respond favorably to targeted therapies, thereby directing treatment strategies (68). However, biomarker profiles and targeted therapies are beyond the scope of this chapter as they play a lesser role in the management of small lung nodules, most of which will be surgically treated.

In a 2017 survey of practice patterns involving Society of Thoracic Radiology members, $60 \%$ of thoracic radiologists reported sending tissue for molecular analysis more than $25 \%$ of the time in cases of suspected lung cancer (26).

As the field of personalized medicine and therapeutics is rapidly evolving there must be strong coordination between the radiologist performing the procedure and the cytologist/ pathologist. Depending on the situation, different amounts of tissue may be necessary. In some cases, when the decision may rest solely on whether a nodule is malignant, then even a single slide may be sufficient for this determination. On the other hand, in certain instances where advanced analyses are necessary several core samples are needed. 
The amount of tissue may also vary depending on available resources within an institution. In some situations, cytology may provide all of the necessary material whereas in others, core samples may be necessary. The critical consideration is that there be good communication both before and after the procedure so that the radiologist fully understands what is needed to be procured so that the necessary analyses can be accomplished. Ideal needle selection for biomarker analysis has been discussed in the Biopsy Planning section of this chapter.

Patients should always be involved and in control of management decisions. Each patient is a unique individual and informed decisions must be elicited.

\section{Conclusion}

In summary, CT-guided biopsy of pulmonary nodules is a well-established, safe, and highly useful diagnostic test. Prebiopsy preparation is a key step in increasing the yield of this procedure. Screening patients for contraindications, coaching the patients throughout the procedure, and carefully planning an ideal needle path are essential for patient safety and procedural success. As CT-guided lung biopsy is highly operator-dependent, developing an expertise in needle steering, becoming familiar with pneumothorax risk factors, and navigating through the optimal needle path will aid in maximizing diagnostic accuracy and reducing complications. The availability of an on-site cytologist or cytotechnologist has been consistently shown to increase diagnostic accuracy. Although many factors such as emphysema severity and nodule location are outside the control of the radiologist, it is important to be prepared for the complications that such factors may bring. As with any procedure, the early recognition and proper management of any biopsy-related complications are a necessary skillset.

\section{Acknowledgments}

Funding: None.

\section{Footnote}

Provenance and Peer Review: This article was commissioned by the Guest Editors (Scott Swanson, Daniel Dolan) for the series "How to Evaluate, Diagnose and Treat Small Lung Nodules" published in Current Challenges in Thoracic Surgery. The article has undergone external peer review.
Peer Review File: Available at https://ccts.amegroups.com/ article/view/10.21037/ccts-21-36/prf

Conflicts of Interest: All authors have completed the ICMJE uniform disclosure form (available at https://ccts.amegroups. com/article/view/10.21037/ccts-21-36/coif). The series "How to Evaluate, Diagnose and Treat Small Lung Nodules" was commissioned by the editorial office without any funding or sponsorship. CIH is a named inventor on a number of patents and patent applications relating to the evaluation of pulmonary nodules on CT scans of the chest which are owned by Cornell Research Foundation (CRF). Since 2009, CIH does not accept any financial benefit from these patents including royalties and any other proceeds related to the patents or patent applications owned by CRF. CIH is the President and serve on the board of the Early Diagnosis and Treatment Research Foundation. She receives no compensation from the Foundation. The Foundation is established to provide grants for projects, conferences, and public databases for research on early diagnosis and treatment of diseases. Recipients include, I-ELCAP, among others. The funding comes from a variety of sources including philanthropic donations, grants and contracts with agencies (federal and non-federal), imaging and pharmaceutical companies relating to image processing assessments. The various sources of funding exclude any funding from tobacco companies or tobaccorelated sources. DFY is a consultant and co-owner of Accumetra, a private company developing tools to improve the quality of CT imaging and is on the medical advisory board of Carestream, a company that develops radiography equipment and has consulted for AstraZeneca, Pfizer and Genentech. In addition, DFY is a named inventor on a number of patents and patent applications related to the evaluation of chest diseases including measurements of chest nodules. DFY has received financial compensation for the licensing of these patents. The authors have no other conflicts of interest to declare.

Ethical Statement: The authors are accountable for all aspects of the work in ensuring that questions related to the accuracy or integrity of any part of the work are appropriately investigated and resolved.

Open Access Statement: This is an Open Access article distributed in accordance with the Creative Commons Attribution-NonCommercial-NoDerivs 4.0 International License (CC BY-NC-ND 4.0), which permits the non- 
commercial replication and distribution of the article with the strict proviso that no changes or edits are made and the original work is properly cited (including links to both the formal publication through the relevant DOI and the license). See: https://creativecommons.org/licenses/by-nc-nd/4.0/.

\section{References}

1. ELCAP Protocol Document. Available online: https:// www.ielcap.org/sites/default/files/I-ELCAP-protocol.pdf Accessed 7/1/2021

2. Lung-RADS Version 1.1. Available online: https://www. acr.org/-/media/ACR/Files/RADS/Lung-RADS/LungRA DSAssessmentCategoriesv1-1.pdf Accessed 7/1/2021

3. MacMahon H, Naidich DP, Goo JM, et al. Guidelines for Management of Incidental Pulmonary Nodules Detected on CT Images: From the Fleischner Society 2017. Radiology 2017;284:228-43.

4. International Early Lung Cancer Action Program Investigators; Henschke CI, Yankelevitz DF, et al. Survival of patients with stage I lung cancer detected on CT screening. N Engl J Med 2006;355:1763-71.

5. Wagnetz U, Menezes RJ, Boerner S, et al. CT screening for lung cancer: implication of lung biopsy recommendations. AJR Am J Roentgenol 2012;198:351-8.

6. Patel IJ, Davidson JC, Nikolic B, et al. Consensus guidelines for periprocedural management of coagulation status and hemostasis risk in percutaneous image-guided interventions. J Vasc Interv Radiol 2012;23:727-36.

7. Manhire A, Charig M, Clelland C, et al. Guidelines for radiologically guided lung biopsy. Thorax 2003;58:920-36.

8. Heerink WJ, de Bock GH, de Jonge GJ, et al. Complication rates of CT-guided transthoracic lung biopsy: meta-analysis. Eur Radiol 2017;27:138-48.

9. Küçük CU, Yilmaz A, Yilmaz A, et al. Computed tomography-guided transthoracic fine-needle aspiration in diagnosis of lung cancer: a comparison of single-pass needle and multiple-pass coaxial needle systems and the value of immediate cytological assessment. Respirology 2004;9:392-6.

10. Geraghty PR, Kee ST, McFarlane G, et al. CT-guided transthoracic needle aspiration biopsy of pulmonary nodules: needle size and pneumothorax rate. Radiology 2003;229:475-81.

11. Yamagami T, Nakamura T, Iida S, et al. Management of pneumothorax after percutaneous CT-guided lung biopsy. Chest 2002;121:1159-64.

12. Ko JP, Shepard JO, Drucker EA, et al. Factors influencing pneumothorax rate at lung biopsy: are dwell time and angle of pleural puncture contributing factors? Radiology 2001;218:491-6.

13. Cox JE, Chiles C, McManus CM, et al. Transthoracic needle aspiration biopsy: variables that affect risk of pneumothorax. Radiology 1999;212:165-8.

14. Laurent F, Michel P, Latrabe V, et al. Pneumothoraces and chest tube placement after CT-guided transthoracic lung biopsy using a coaxial technique: incidence and risk factors. AJR Am J Roentgenol 1999;172:1049-53.

15. García-Río F, Pino JM, Casadevall J, et al. Use of spirometry to predict risk of pneumothorax in CT-guided needle biopsy of the lung. J Comput Assist Tomogr 1996;20:20-3.

16. Li H, Boiselle PM, Shepard JO, et al. Diagnostic accuracy and safety of CT-guided percutaneous needle aspiration biopsy of the lung: comparison of small and large pulmonary nodules. AJR Am J Roentgenol 1996;167:105-9.

17. Kazerooni EA, Lim FT, Mikhail A, et al. Risk of pneumothorax in CT-guided transthoracic needle aspiration biopsy of the lung. Radiology 1996;198:371-5.

18. Fish GD, Stanley JH, Miller KS, et al. Postbiopsy pneumothorax: estimating the risk by chest radiography and pulmonary function tests. AJR Am J Roentgenol 1988;150:71-4.

19. Poe RH, Kallay MC, Wicks CM, et al. Predicting risk of pneumothorax in needle biopsy of the lung. Chest 1984;85:232-5.

20. Ozturk K, Soylu E, Gokalp G, et al. Risk factors of pneumothorax and chest tube placement after computed tomography-guided core needle biopsy of lung lesions: a single-centre experience with 822 biopsies. Pol J Radiol 2018;83:e407-14.

21. Kuban JD, Tam AL, Huang SY, et al. The Effect of Needle Gauge on the Risk of Pneumothorax and Chest Tube Placement After Percutaneous Computed Tomographic (CT)-Guided Lung Biopsy. Cardiovasc Intervent Radiol 2015;38:1595-602.

22. Nour-Eldin NE, Alsubhi M, Emam A, et al. Pneumothorax Complicating Coaxial and Non-coaxial CT-Guided Lung Biopsy: Comparative Analysis of Determining Risk Factors and Management of Pneumothorax in a Retrospective Review of 650 Patients. Cardiovasc Intervent Radiol 2016;39:261-70.

23. Nour-Eldin NE, Alsubhi M, Naguib NN, et al. Risk factor analysis of pulmonary hemorrhage complicating CTguided lung biopsy in coaxial and non-coaxial core biopsy 
techniques in 650 patients. Eur J Radiol 2014;83:1945-52.

24. Aviram G, Schwartz DS, Meirsdorf S, et al. Transthoracic needle biopsy of lung masses: a survey of techniques. Clin Radiol 2005;60:370-4.

25. Chen L, Jing H, Gong Y, et al. Diagnostic efficacy and molecular testing by combined fine-needle aspiration and core needle biopsy in patients with a lung nodule. Cancer Cytopathol 2020;128:201-6.

26. Lee C, Guichet PL, Abtin F. Percutaneous Lung Biopsy in the Molecular Profiling Era: A Survey of Current Practices. J Thorac Imaging 2017;32:63-7.

27. Yankelevitz DF, Henschke CI, Koizumi JH, et al. CTguided transthoracic needle biopsy of small solitary pulmonary nodules. Clin Imaging 1997;21:107-10.

28. Yankelevitz DF, Davis SD, Chiarella D, et al. Needletip repositioning during computed-tomographyguided transthoracic needle aspiration biopsy of small deep pulmonary lesions: minor adjustments make a big difference. J Thorac Imaging 1996;11:279-82.

29. Li Y, Du Y, Yang HF, et al. CT-guided percutaneous core needle biopsy for small $(\leq 20 \mathrm{~mm})$ pulmonary lesions. Clin Radiol 2013;68:e43-8.

30. Adams J, Wu HH. The utility of fine-needle aspiration in the diagnosis of primary and metastatic tumors to the lung: a retrospective examination of 1,032 cases. Acta Cytol 2012;56:590-5.

31. Khorsandi M, Shaikhrezai K, Wallace W, et al. Is fineneedle aspiration diagnosis of malignancy adequate prior to major lung resections including pneumonectomy? Interact Cardiovasc Thorac Surg 2012;15:253-7.

32. Lopes Pegna A, Picozzi G, Falaschi F, et al. Four-year results of low-dose CT screening and nodule management in the ITALUNG trial. J Thorac Oncol 2013;8:866-75.

33. Suresh S, Salama GR, Ramjit A, et al. CT-Guided FineNeedle Aspiration Biopsy of Pulmonary Nodules 8 mm or Less Has a Higher Diagnostic Accuracy than Positron Emission Tomography-CT. J Vasc Interv Radiol 2018;29:520-3.

34. Zhao Y, Matsui Y, Hiraki T, et al. Computed tomography fluoroscopy-guided cutting needle biopsy of pulmonary nodules $\leq 8 \mathrm{~mm}$ : A retrospective study including 117 nodules. Eur J Radiol 2020;125:108903.

35. Chang YY, Chen CK, Yeh YC, et al. Diagnostic feasibility and safety of CT-guided core biopsy for lung nodules less than or equal to $8 \mathrm{~mm}$ : A single-institution experience. Eur Radiol 2018;28:796-806.

36. Li Y, Wang T, Fu YF, et al. Computed tomography-guided biopsy for sub-centimetre lung nodules: Technical success and diagnostic accuracy. Clin Respir J 2020;14:605-10.

37. Huang MD, Weng HH, Hsu SL, et al. Accuracy and complications of CT-guided pulmonary core biopsy in small nodules: a single-center experience. Cancer Imaging 2019;19:51.

38. Chen C, Xu L, Sun X, et al. Safety and diagnostic accuracy of percutaneous CT-guided transthoracic biopsy of small lung nodules $(\leq 20 \mathrm{~mm})$ adjacent to the pericardium or great vessels. Diagn Interv Radiol 2021;27:94-101.

39. Gelbman BD, Cham MD, Kim W, et al. Radiographic and clinical characterization of false negative results from CTguided needle biopsies of lung nodules. J Thorac Oncol 2012;7:815-20.

40. Uzun Ç, Akkaya Z, Düşünceli Atman E, et al. Diagnostic accuracy and safety of CT-guided fine needle aspiration biopsy of pulmonary lesions with non-coaxial technique: a single center experience with 442 biopsies. Diagn Interv Radiol 2017;23:137-43.

41. Maxwell AW, Klein JS, Dantey K, et al. CT-guided transthoracic needle aspiration biopsy of subsolid lung lesions. J Vasc Interv Radiol 2014;25(3):340-6, 346.e1.

42. De Filippo M, Saba L, Concari G, et al. Predictive factors of diagnostic accuracy of CT-guided transthoracic fineneedle aspiration for solid noncalcified, subsolid and mixed pulmonary nodules. Radiol Med 2013;118:1071-81.

43. Yun S, Kang H, Park S, et al. Diagnostic accuracy and complications of CT-guided core needle lung biopsy of solid and part-solid lesions. Br J Radiol 2018;91:20170946.

44. Kiranantawat N, McDermott S, Petranovic M, et al. Determining malignancy in CT-guided fine needle aspirate biopsy of subsolid lung nodules: Is core biopsy necessary? Eur J Radiol Open 2019;6:175-81.

45. Ohno Y, Hatabu H, Takenaka D, et al. CT-guided transthoracic needle aspiration biopsy of small $(<$ or $=20$ $\mathrm{mm}$ ) solitary pulmonary nodules. AJR Am J Roentgenol 2003;180:1665-9.

46. Nakamura M, Yoshizako T, Koyama S, et al. Risk factors influencing chest tube placement among patients with pneumothorax because of CT-guided needle biopsy of the lung. J Med Imaging Radiat Oncol 2011;55:474-8.

47. Lendeckel D, Kromrey ML, Ittermann T, et al. Pulmonary emphysema is a predictor of pneumothorax after CTguided transthoracic pulmonary biopsies of pulmonary nodules. PLoS One 2017;12:e0178078.

48. Lee KH, Lim KY, Suh YJ, et al. Nondiagnostic Percutaneous Transthoracic Needle Biopsy of Lung Lesions: A Multicenter Study of Malignancy Risk. Radiology 2019;290:814-23. 
49. Yankelevitz DF, Davis SD, Henschke CI. Aspiration of a large pneumothorax resulting from transthoracic needle biopsy. Radiology 1996;200:695-7.

50. Yamagami T, Kato T, Hirota T, et al. Usefulness and limitation of manual aspiration immediately after pneumothorax complicating interventional radiological procedures with the transthoracic approach. Cardiovasc Intervent Radiol 2006;29:1027-33.

51. Moore EH. Technical aspects of needle aspiration lung biopsy: a personal perspective. Radiology 1998;208:303-18.

52. Graffy P, Loomis SB, Pickhardt PJ, et al. Pulmonary Intraparenchymal Blood Patching Decreases the Rate of Pneumothorax-Related Complications following Percutaneous CT-Guided Needle Biopsy. J Vasc Interv Radiol 2017;28:608-613.e1.

53. Wagner JM, Hinshaw JL, Lubner MG, et al. CT-guided lung biopsies: pleural blood patching reduces the rate of chest tube placement for postbiopsy pneumothorax. AJR Am J Roentgenol 2011;197:783-8.

54. Li Y, Du Y, Luo TY, et al. Usefulness of normal saline for sealing the needle track after CT-guided lung biopsy. Clin Radiol 2015;70:1192-7.

55. Ahrar JU, Gupta S, Ensor JE, et al. Efficacy of a Selfexpanding Tract Sealant Device in the Reduction of Pneumothorax and Chest Tube Placement Rates After Percutaneous Lung Biopsy: A Matched Controlled Study Using Propensity Score Analysis. Cardiovasc Intervent Radiol 2017;40:270-6.

56. Huo YR, Chan MV, Habib AR, et al. Post-Biopsy Manoeuvres to Reduce Pneumothorax Incidence in CTGuided Transthoracic Lung Biopsies: A Systematic Review and Meta-analysis. Cardiovasc Intervent Radiol 2019;42:1062-72.

57. Maybody M, Muallem N, Brown KT, et al. Autologous Blood Patch Injection versus Hydrogel Plug in CT-guided Lung Biopsy: A Prospective Randomized Trial. Radiology 2019;290:547-54.

58. Ramaswamy R, Narsinh KH, Tuan A, et al. Systemic Air

doi: $10.21037 /$ ccts-21-36

Cite this article as: Cham MD, Henschke CI, Yankelevitz DF. The radiologist's role in pathologic diagnosis of small lung nodules: radiographic methods of tissue acquisition. Curr Chall Thorac Surg 2022;4:33.
Embolism following Percutaneous Lung Biopsy. Semin Intervent Radiol 2014;31:375-7.

59. Byrd RP Jr, Fields-Ossorio C, Roy TM. Delayed chest radiographs and the diagnosis of pneumothorax following CT-guided fine needle aspiration of pulmonary lesions. Respir Med 1999;93:379-81.

60. Choi CM, Um SW, Yoo CG, et al. Incidence and risk factors of delayed pneumothorax after transthoracic needle biopsy of the lung. Chest 2004;126:1516-21.

61. Traill ZC, Gleeson FV. Delayed pneumothorax after CTguided percutaneous fine needle aspiration lung biopsy. Thorax 1997;52:581-2; discussion 575-6.

62. Dennie CJ, Matzinger FR, Marriner JR, et al. Transthoracic needle biopsy of the lung: results of early discharge in 506 outpatients. Radiology 2001;219:247-51.

63. Pua U, Quek LH, Lim GH, et al. Single-Session Multilevel "Central Stentoplasty". J Vasc Interv Radiol 2016;27:416-7.

64. Austin JH, Cohen MB. Value of having a cytopathologist present during percutaneous fine-needle aspiration biopsy of lung: report of 55 cancer patients and metaanalysis of the literature. AJR Am J Roentgenol 1993;160:175-7.

65. Santambrogio L, Nosotti M, Bellaviti N, et al. CT-guided fine-needle aspiration cytology of solitary pulmonary nodules: a prospective, randomized study of immediate cytologic evaluation. Chest 1997;112:423-5.

66. Tachibana K, Nakazato Y, Tsuchida S, et al. Immediate cytology improves accuracy and decreases complication rate in real-time computed tomography-guided needle lung biopsy. Diagn Cytopathol 2013;41:1063-8.

67. Zudaire I, Lozano MD, Vazquez MF, et al. Molecular characterization of small peripheral lung tumors based on the analysis of fine needle aspirates. Histol Histopathol 2008;23:33-40.

68. Mendoza DP, Piotrowska Z, Lennerz JK, et al. Role of imaging biomarkers in mutation-driven non-small cell lung cancer. World J Clin Oncol 2020;11:412-27. 